\section{REVIEW ARTICLE}

\title{
Transcription factors in Candida albicans - environmental control of morphogenesis
}

\author{
Joachim F. Ernst \\ Tel: +49211811 5176. Fax: +49211811 5176. e-mail: joachim.ernst@uni-duesseldorf.de
}

Institut für Mikrobiologie, Heinrich-Heine-Universität, Universitätsstr. 1/26.12, D-40225 Düsseldorf, Germany

Keywords: Candida, morphogenesis, dimorphism, transcription factors, signalling

\section{Overview}

Awareness of Candida albicans as a major human health threat has risen during recent years. Although infections by $C$. albicans can be relatively mild and superficial, systemic mycoses often occur in immunocompromised patients, or even as a consequence of long-term therapy with broad-spectrum antibiotics or of chemotherapy (reviewed by Odds, 1988). Effective antifungal agents which are free of side-effects are urgently needed. There is hope that recently developed techniques of manipulating $C$. albicans and the sequencing of its genome will lead to a thorough understanding of the virulence and biology of this fungal pathogen, thus offering the possibility of a knowledge-based approach to novel antifungal agents.

Experimental work on C. albicans has long been hampered by its asexuality, its diploidy and its noncanonical codon usage (CUG encodes serine) (reviewed by Scherer \& Magee, 1990; Santos et al., 1996). Molecular-genetic techniques have overcome these difficulties, and today host strains and transformation vectors are available and an efficient gene-disruption protocol has been developed (Fonzi \& Irwin, 1993; Morschhäuser et al., 1999; Wilson et al., 1999). Reporter genes encoding $\beta$-galactosidase (Lac4p), luciferase and green fluorescent protein (GFP) are available (Leuker et al., 1992; Srikantha et al., 1996; Cormack et al., 1997). The Stanford genome-sequencing project is expected to complete 10 -fold coverage of the $C$. albicans genome in the year 2000 (http://www-sequence.stanford.edu/ group/candida/).

Which factors determine the virulence of C. albicans? In the past possible virulence traits have been suggested, which were characterized on a phenomenological level, but studies could not prove their role in pathogenesis. Modern techniques allow the testing of such hypotheses. It is generally accepted now that dimorphism, i.e. the ability to grow in a yeast and a filamentous growth form, not only is a virulence trait per se, but is also coregulated with other virulence factors, which are associated with cellular morphology (Hube et al., 1996;
Lo et al., 1997). Dimorphism and other striking morphogenetic phenotypes of C. albicans, including chlamydospore formation and phenotypic switching, are regulated by environmental conditions. This review focuses on transcription factors that mediate environmental control and thereby constitute key elements of morphogenesis in C. albicans.

\section{A variety of developmental programmes}

C. albicans is able to develop single spherical cells including typical yeast cells and chlamydospores, as well as elongated cells developing into multicellular true hyphae or pseudohyphae (Fig. 1). Thus, the term dimorphism, which traditionally is reserved for the yeast-true hypha interconversion, in a more general sense designates the main theme of C. albicans and possibly fungal morphogenesis in general. Buddingyeast cells can be induced to form true hyphae, which grow by continuous apical extension followed by septation. Pseudohyphae grow differently from true hyphae, by unipolar budding: buds develop into elongated cells, which remain attached to mother cells, stop growth and resume budding. Chlamydospores are thickwalled spherical cells and develop on pseudohyphal support cells (Joshi et al., 1993; Montazeri \& Hedrick, 1984). Finally, in some forms of spontaneous phenotypic switching (reviewed by Soll et al., 1993; Soll, 1997), enlarged elongated cells arise, which have a different appearance to yeast and pseudohyphal cells, and which do not form extensive filaments (opaque phenotype) (Fig. 1).

Dimorphic fungi share cell morphologies with both filamentous fungi and yeasts. This raises the question whether in evolution an ancestral yeast-like species has gained the ability to develop hyphae or conversely, whether a primordial filament-forming fungus has acquired the ability to develop a yeast form. The answer to this question is unknown, but some hints favour the latter possibility. Firstly, the position of hypha (germ tube) development on a C. albicans yeast mother cell is random with respect to previous sites of budding 


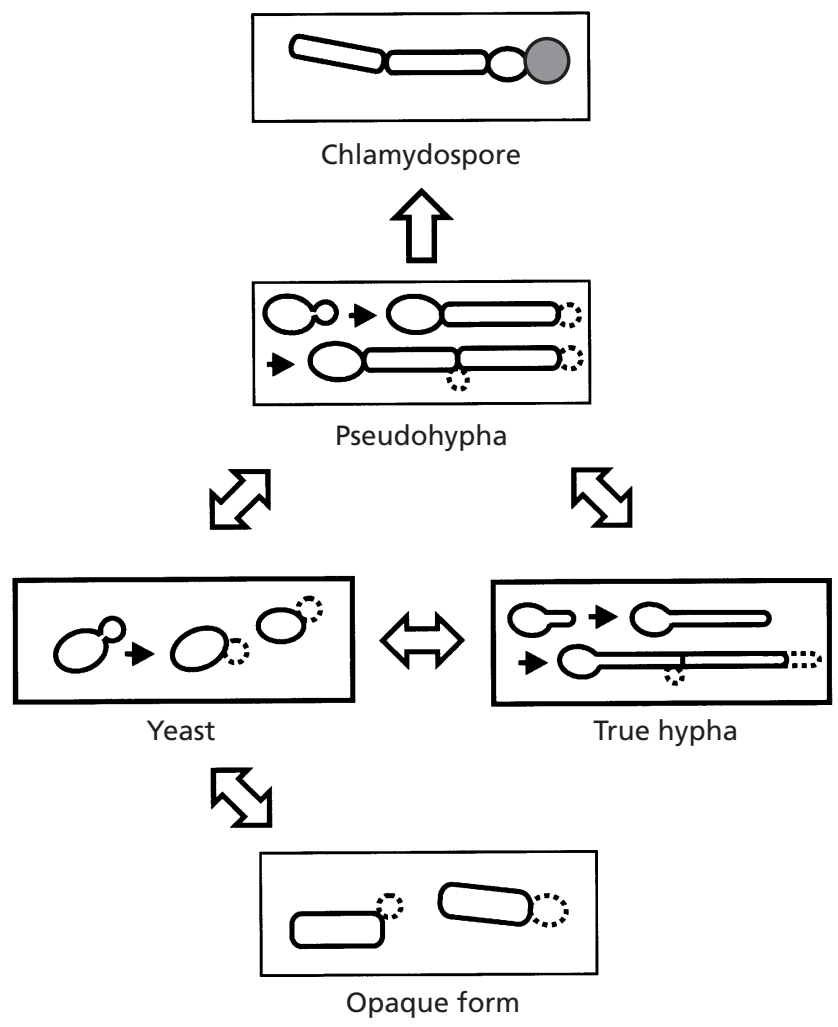

Fig. 1. Morphological forms of Candida albicans. The yeast and hyphal growth forms are predominant during infection of the human host. Chlamydospores are thick-walled spherical cells arising as terminal cells on pseudohyphae; pathways of chlamydospore germination are unknown. Daughter cells and regions of cell expansion are indicated by dotted lines.

(Chaffin, 1984) and thus is similar to Saccharomyces cerevisiae yeast mutants, which lack components for the positioning of polar and axial buds (Chant, 1994). Secondly, several mutations of C. albicans discussed below (efg1, tup1, rbf1) favour an elongated cell morphology suggesting that filamentation is under negative control. If this hypothesis is correct, 'add-on' components must have evolved to repress filamentation and to favour spherical cell growth in an ancestral yeastlike organism.

\section{Environmental cues regulate transcription factors}

C. albicans morphology is directly related to environmental conditions. Hyphal development depends on two factors: (1) the nature, number and intensity of environmental signals (outside cues) and (2) the activity of signalling pathways including key transcription factors (cellular-response machinery). According to current models, environmental cues trigger separate signal-transduction pathways, which regulate common targets required to initiate hyphal growth (Fig. 2). A threshold level of signalling may be reached either by simultaneous low-level stimulation of several pathways or by essentially a single pathway, which is fully activated (or hyperactive as in overexpression experiments). The most powerful hypha-inducing protocol involves incubation of C. albicans at low cell densities in liquid medium containing serum, GlcNAc or other inducers, at $37^{\circ} \mathrm{C}$ (Shepherd et al., 1980). Conceivably, because of strong stimulation of several pathways, minor defects in signalling pathways are not detected under this condition. Liquid induction is usually monitored for a few hours, when initial germ tubes have developed into hyphae that begin to bud off yeast cells. In contrast, on solid media cells develop into colonies over several days of growth, until (pseudo-) hyphal growth emerging from the colonies is recorded. The latter procedure is somewhat problematic, because (1) the molecular environment of a fully developed colony will differ greatly from the initial media composition, not allowing a clear definition of the inducing environment - in fact colonies 'condition' their environment by depletion of nutrients and by secretion of fungal compounds (Ritz \& Crawford, 1999) and (2) cells may filament efficiently during initial growth, but may revert quickly to yeast growth and the phenotype of a grown colony will be determined by not only the yeast-hypha transition, but also the reverse process. We found that the use of $1 \%$ proline as a single carbon and nitrogen source in solid media strongly induced filaments until microcolonies appeared, but growth later completely reverted to the yeast form, leading to a yeast-only appearance after several days (Ernst, 2000). The 'switch back' to yeast growth is delayed in cpp1 mutants (Csank et al., 1997) or by overexpression of the TPK2 gene (Sonneborn et al., 2000), while it occurs more rapidly in cln1 mutants (Loeb et al., 1999). Compared to liquid medium, induction on solid media appears to represent a weaker hypha-inducing condition, because minor defects in filamentation (as in many mutants, which do not possess the full repertoire of signalling pathways; see below) show a defective phenotype on solid but not in liquid media.

\section{Serum and other inducers}

Serum of different sources is still the 'magic potion' to rapidly induce true hyphae in $C$. albicans. The responsible factor is not albumin, since albumin-free serum from a rat mutant was as efficient at promoting hyphae as normal serum (Feng et al., 1999). Two known inducers of hypha formation, $\mathrm{N}$-acetylglucosamine (GlcNAc) and proline, may contribute to the serum effect since they are generated by degradation of serum (glyco-) proteins. Thus, several independent signalling pathways are likely to be triggered by serum. Hyphae arise within minutes at $37^{\circ} \mathrm{C}$ at $5-20 \%$ serum in liquid; on solid media hyphae development is triggered within a few hours. Numerous other compounds induce hyphae in vitro (reviewed by Odds, 1988), but their significance for the infection process is unknown.

The Efg1 protein is a strong regulator of morphogenetic processes in C. albicans, since it influences not only yeast-hypha interconversions (Stoldt et al., 1997; Lo et 


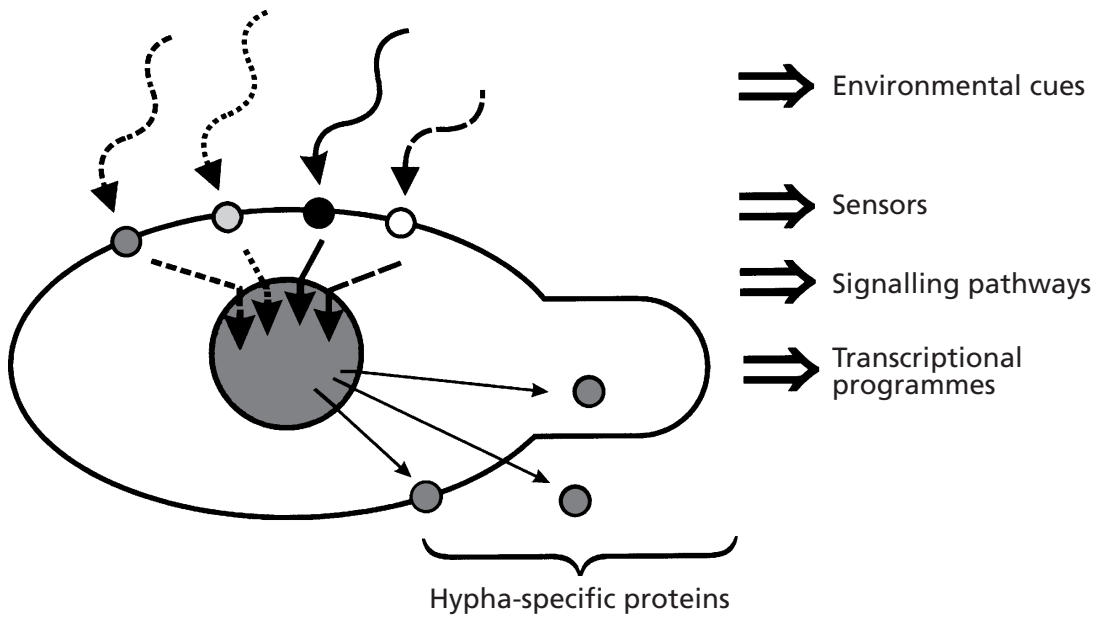

Fig. 2. General scheme of hypha induction. Various environmental cues activate separate signalling pathways via sensor proteins. Signalling pathways may converge on separate or identical transcription factors and transcription factors may converge on common target genes to trigger expression of hypha-specific genes. al., 1997), but also regulates phenotypic switching and chlamydospore formation of this pathogen (Sonneborn et al., 1999a, b) (Fig. 3). The role of Efg1p in hyphal morphogenesis is fascinating, because under standard induction conditions, using serum or GlcNAc as inducers in liquid or on solid media, there is essentially a complete block of hyphal formation in strains lacking Efg1p (Stoldt et al., 1997; Lo et al., 1997). On the other hand, under microaerophilic/embedded conditions hyphal formation is not defective at all in homozygous efg1 mutants, but rather appears stimulated (Sonneborn et al., 1999b; Riggle et al., 1999; Brown et al., 1999). To make things more complicated, the lack of EFG1 expression in strains CAI8 and a WO-1 derivative (but not in the common strain CAI4) favours a rod-like, pseudohyphal phenotype (Sonneborn et al., 1999a; Srikantha et al., 2000). These results indicate that, depending on environmental cues (and depending on the genetic background), Efg1p is an activator and repressor of morphogenesis. Efg1p is a member of the APSES protein group, which encompasses putative transcription factors involved in fungal morphogenetic processes leading to (1) a transition from tubular to spherical growth such as in the case of the Asm 1 protein of Neurospora crassa, which is involved in ascospore maturation (Aramayo et al., 1996) or the StuA protein of Aspergillus nidulans, which is required for conidiophore formation (Miller et al., 1992), or (2) regulation of the transition from spherical growth to tubular growth, such as the yeast-filament transition, which is regulated by the Phd 1 and Sok 2 proteins of S. cerevisiae (Gimeno \& Fink, 1994; Ward et al., 1995). Efg1p has both types of activities, since it is required for the yeasthyphal transition, as well as for the pseudohyphachlamydospore morphogenesis (Stoldt et al., 1997; Lo et al., 1997; Sonneborn et al., 1999b). APSES proteins share $80-90 \%$ identical residues in a domain encompassing about $100 \mathrm{aa}$. We discovered that the conserved domain of APSES proteins contains a basic helixloop-helix (bHLH) motif, which is known to be required for dimerization and DNA binding (Stoldt et al., 1997); this theory was supported later by molecular modelling (Dutton et al., 1997). However, because the Phd1 and Sok2 proteins have opposite functions, as activator and repressor, respectively, the conserved APSES domain is not sufficient to determine the direction of morphogenesis. Auxiliary proteins are likely to determine the specific functions of Efg1p, as in the case of the well-studied Myc protein, whose action as an activator or repressor is determined by interacting proteins (reviewed by Bernards, 1995). The presumed bHLH motif in Efg1p indicates an E box binding site on promoter regions of target genes (CANNTG). However, genes may be regulated by bHLH proteins, even if they do not contain an $\mathrm{E}$ box, as shown for the autoregulation of the Myc protein (Facchini et al., 1997). We have recently shown that the EFG1 gene is also autoregulated (B. Tebarth, M. Weide \& J. F. Ernst, unpublished).

Added cAMP has been reported to induce hyphae in $C$. albicans (Niimi et al., 1980; Sabie \& Gadd, 1992; Niimi, 1996), suggesting that a cAMP pathway controls hyphal morphogenesis as it controls pseudohyphal development in S. cerevisiae (Gimeno et al., 1992; Mösch et al., 1996; Robertson \& Fink, 1998; Pan \& Heitman, 1999). Recently, RAS1 has been characterized, which appears to be the only RAS gene in C. albicans (Feng et al., 1999). Homozygous ras1 mutants are unable to form true hyphae in the presence of serum, while pseudohyphae are still observed. A dominant-active version of Ras1p stimulates hyphal growth, while a dominant-negative version represses it. Recently, the TPK2 gene encoding the catalytic subunit of a protein kinase A (PKA) isoform has been characterized (homologue of $S$. cerevisiae TPK2) (Sonneborn et al., 2000), which conceptually could function downstream of Ras1p and be regulated by cAMP levels. Hyphal morphogenesis of a homozygous tpk2 mutant is blocked on solid media containing serum and starvation-type (Spider) medium; interestingly, in liquid a defect is hardly seen with serum as the inducer, while it is apparent in Spider medium. The defects are especially pronounced at $30^{\circ} \mathrm{C}$ and are less detectable at $37^{\circ} \mathrm{C}$. Thus, if relatively weak environmental signals (low temperature, solid medium, 


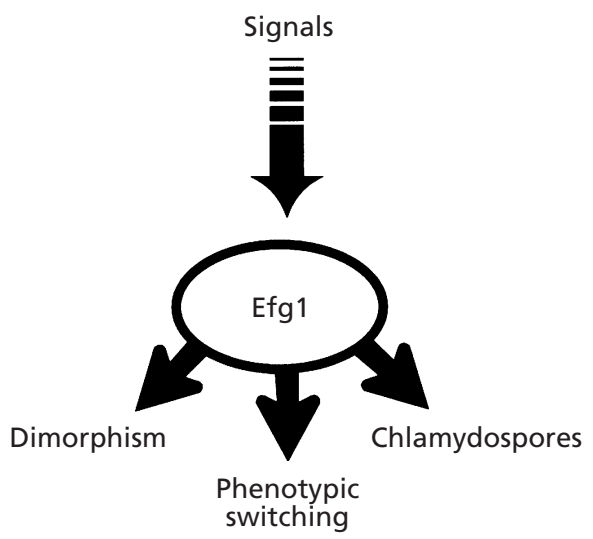

Fig. 3. Various roles of the Efg1 morphogenetic regulator. In dimorphism Efg1p is an activator of hypha formation under some conditions (serum, GIcNAc induction), while under other conditions (embedded/microaerophilic) it is not required and possibly acts as a repressor. Chlamydospore formation and the phenotypic switching from the opaque to the white (yeast) phase phenotype requires Efg1p.

Spider medium) encounter a defective response machinery, the result is a defective phenotype, while there is hardly a phenotype under optimal induction conditions, because additional pathways compensate for the defect. The partial effect of the tpk2 mutation compared to the ras1 mutation may be due to a second PKA isoform (Tpk1p), which is indicated by the Candida genome-sequencing project (http://wwwsequence.stanford.edu/group/candida/). The tpk2 morphogenetic phenotype is suppressed by overexpression of EFG1, while the reverse is not true indicating that Efg1p is a downstream target of Tpk2p. We recently pointed out that Efg1p contains only a single potential site of phosphorylation by PKA (T206) (Sonneborn et al., 1999b, 2000), at a similar position to other APSES proteins, with the exception of Phd1p (in this respect Sok2p appears more similar to Efg1p than Phd1p). Cells producing an Efg1p mutant version containing the T206A mutation are partially blocked in hyphal formation, while the T206E variant is hyperfilamentous (D. Bockmühl, A. Sonneborn \& J. F. Ernst, unpublished). Taken together, these results suggest that Efg1p may be the constituent of a Ras1/cAMP/PKA pathway. Indirect data support the presence of PKA and APSES proteins in common signalling pathways in fungi. A heterologous C. albicans chaperonin subunit (Cct8p) blocked pseudohyphal growth in $S$. cerevisiae, if morphogenesis was induced by Ras $2 \mathrm{p}$, by Phd1p and the heterologous Efg1 protein, but not if it was triggered by activation of the MAP kinase pathway. In addition, morphogenesis-unrelated Ras2 phenotypes were blocked, suggesting PKA as the target of suppression (Rademacher et al., 1998). We suggested that extraneous Cct8 protein could sequester, but not fold, PKA isoforms, thereby lowering PKA activity levels. These results are consistent with the presence of PKA and APSES proteins in a common pathway (Fig. 4).

\section{Starvation}

In $S$. cerevisiae nitrogen limitation stimulates pseudohyphal growth (Gimeno et al., 1992). This morphogenesis is not detected in liquid medium, but only on solid nitrogen-limiting (SLAD) medium; here a yeast colony first grows to a considerable size within several days before lateral pseudohyphae appear. C. albicans is also triggered on SLAD medium to form filaments (true hyphae) after several days growth (Csank et al., 1998). A similar phenotype occurs on a medium containing mannitol as a source of carbon and a complex source of nitrogen (modified Lee's medium or Spider medium) (Liu et al., 1994). The late development of hyphae on these media suggests that some media component has to be consumed before filamentation is triggered. It has been known for a long time that pregrowth of cells into stationary phase or a period of starvation enhances the efficiency of subsequent hyphal induction by inducers like serum or GlcNAc (Shepherd et al., 1980; Delbrück \& Ernst, 1993). The addition of ammonium salts to Spider medium did not block hyphal development, indicating that nitrogen limitation is not responsible for filamentation in this medium (Ernst, 2000).

Nitrogen starvation in $S$. cerevisiae activates a relay of kinases, including Ste20p, Ste7p and the mitogenactivated protein (MAP) kinase Kss1p, to ultimately phosphorylate the transcription factor Ste12p (Liu et al., 1993; Madhani \& Fink, 1997). Homologous kinases (Hst11p, Hst7p, Cek1p) exist in C. albicans, along with the Ste12p homologue Cph1p; epistasis experiments have ordered most of these components in a linear pathway (Liu et al., 1994; Köhler \& Fink, 1996; Leberer et al., 1996; Csank et al., 1998) (Fig. 4). While mutations in the MAP kinase cascade efficiently block pseudohyphal development in S. cerevisiae, hyphal morphogenesis in C. albicans is blocked only on certain solid starvation-type (Spider or SLAD) media (Liu et al., 1994; Köhler \& Fink, 1996; Leberer et al., 1996; Csank et al., 1998). On other solid media and generally in liquid media these mutations do not affect hyphal growth. Virulence of cst20, hst7 and cph1 mutants is partially affected in the mouse model of systemic candidiasis. It is occasionally stated in the literature that both $c p h 1$ and efg1 mutants are partially defective in hyphal development and that the combination of both mutations blocks hyphal growth. This statement is misleading, because (true) hypha formation in efg1 mutant is almost completely blocked under all standard induction conditions (Stoldt et al., 1997; Lo et al., 1997; Ernst, 2000) and the presence of a cph1 mutation does not contribute to the (true) hypha-negative phenotype of a efg1 mutant (although pseudohypha formation is lower in a efg1 $c p h 1$ double mutant compared to an efg1 mutant). A minor contribution of the cph1 mutation to hyphal growth was detected by comparing homozygous tup1 efg1 and tup1 efg1 cph1 mutants (Braun \& Johnson, 2000). In addition, the $c p h 1$ mutation also contributed to the defective phenotype of $c z f 1$ mutants under microaerophilic/embedded conditions in starvationtype media (see below) (Brown et al., 1999). 


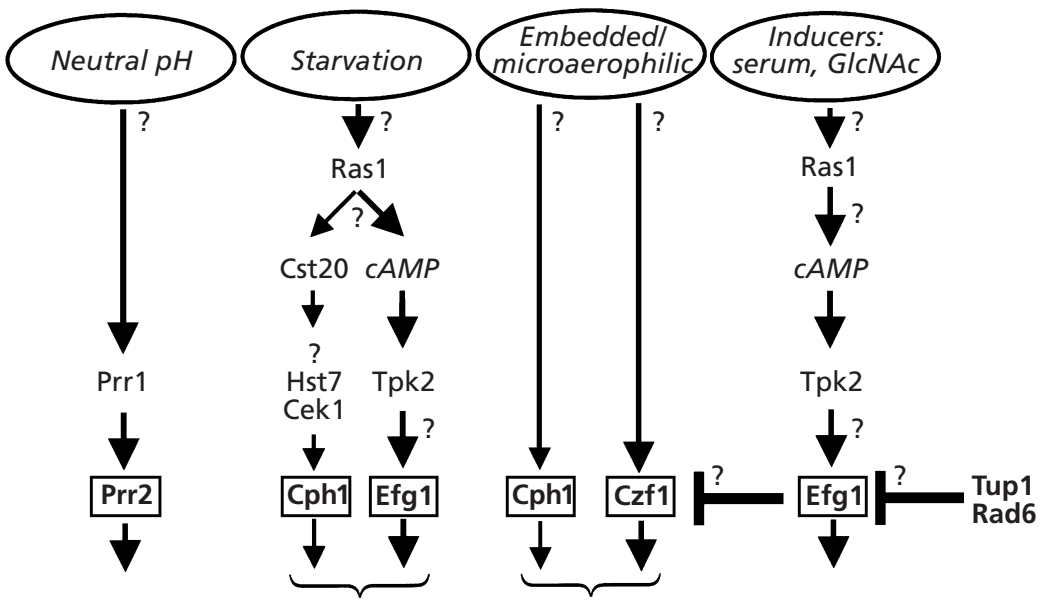

True hypha
Fig. 4. Environmental cues stimulate separate signalling pathways. There is no unitary signalling pathway leading to (true) hyphal formation. Different combinations of pathways, at varying intensities, are triggered in different environments. Transcription factors activated by the respective pathways are boxed. Pathways leading to pseudohypha formation are presumed to be different from the pathways triggering formation of true hyphae (not shown).
Hyperactivation of the MAP kinase pathway leads to filamentation on starvation-type media and has been achieved by overexpression of components of the MAP kinase pathway (Leberer et al., 1996; Csank et al., 1998). The cpp1 mutation also stimulates this pathway, presumably because the Cpp1 protein is a member of the VH1 family of dual-specificity phosphatases, whose function could be to dephosphorylate and thereby inactivate the Cek1p MAP kinase (Csank et al., 1997). In vitro inactivation of mammalian MAP kinases, its tyrosine-phosphate specificity and the reversal of the $c p p 1$ mutant phenotype by a cek1 mutation support this function of Cpp1p. Hyperactivation of the MAP kinase pathway is also achieved by deletion of the HOG1 gene (Alonso-Monge et al., 1999) (see below).

\section{Embedded/microaerophilic conditions}

Although homozygous efg1 single mutants have a drastic block in true hyphal formation under most standard induction conditions, considerable filamentation occurs in certain other environments (Brown et al., 1999; Sonneborn et al., 1999b). A limited supply of oxygen, as occurs under a coverslip during induction of chlamydospores, allows wild-type cells to form filaments, which is enhanced in efg1 mutants (Sonneborn et al., 1999b). Similarly, growth of wild-type colonies embedded in agar stimulates filamentation, which still occurs in homozygous efg1 cph1 mutants (Riggle et al., 1999; A. Giusani \& C. Kumamoto, unpublished). Thus, there appears to exist an Efg1p-independent pathway of filamentation in C. albicans, which is operative under microaerophilic/embedded conditions. The filaments produced under microaerophilic conditions have the characteristics of mostly pseudohyphae in EFG1 wildtype strains and of mostly true hyphae in efg1 mutants (Sonneborn et al., 1999b); under embedded conditions mostly true hyphae were produced (Brown et al., 1999). Interestingly, the alternative filamentation pathway not only is independent of Efg $1 \mathrm{p}$, but it is even repressed by it to some degree. The enhanced filamentation in efg1 mutants does not depend on the Cph1 MAP kinase, because a homozygous efg1 cph1 strain is as hyperfilamentous as the homozygous efg1 mutant (Sonneborn et al., 1999b). It is possible that agar embedding generates microaerophilic conditions, which activate the same Efg1p-independent pathway of morphogenesis under both conditions (Fig. 4).

The putative transcription factor Czf1 is probably an important element of the alternative pathway of filamentation in C. albicans (Brown et al., 1999). The central portion of Czf1p contains four clusters of glutamine residues and the $\mathrm{C}$ terminus contains a cysteine-rich region similar to zinc-finger elements. There is no direct homologue of Czf1p in the genome of $S$. cerevisiae. Overexpression of CZF1 stimulates filamentous growth, but only under embedded conditions and in certain media lacking glucose. Homozygous czf1 null mutants filament normally under standard induction conditions, but they are defective in hyphal development when embedded in agar. This defective phenotype occurs only during embedding in certain media, such as complex medium containing sucrose or galactose as carbon sources at $25^{\circ} \mathrm{C}$, but not at $37^{\circ} \mathrm{C}$ or in media containing strong inducers including serum and GlcNAc. These characteristics suggest that factors other than Czf1p contribute to filamentation under embedded conditions. The defective phenotype of a $c z f 1$ mutant is exacerbated by the presence of a $c p h 1$ mutation, which by itself shows defects in the types of media used for monitoring the $c z f 1$ phenotype. Thus, although the $c p h 1$ mutant phenotype does not appear to be specific for embedded conditions, it worsens filamentation defects caused by the $c z f 1$ mutation.

Hyperfilamentation of efg1 single and $e f g 1 c p h 1$ double mutants suggests that Efg1p is a negative modulator of the Czf1p pathway under microaerophilic/embedded conditions (Fig. 4). Thus, these data once again suggest that positive and negative functions are combined in the Efg1 protein. Conceivably, Efg1p and Czf1p collaborate 
to allow filamentation in different host environments, as in the blood (serum) and during the passage of tissues or within host cells, at low oxygen partial pressure. It is also possible that Efg1p and Czf1p trigger the formation of different types of hyphae, each of which are equipped with different sets of proteins required for viability and virulence in specific host niches, such as the blood and at limiting oxygen concentrations within cells or within organs.

\section{pH}

It is well established that a $\mathrm{pH}$ around neutrality $(\mathrm{pH}$ $\sim 6.5$ ) favours hyphal development of C. albicans in vitro, while a low $\mathrm{pH}(\mathrm{pH}<6.5)$ blocks hyphal formation and stimulates growth of the yeast form (Buffo et al., 1984). The Prr2 transcription factor, which has homologues in other fungi, has a central role in $\mathrm{pH}$-dependent regulation by inducing the expression of alkaline-expressed genes and repressing acid-expressed genes at alkaline $\mathrm{pH}$ (Ramon et al., 1999). prr2 mutants are unable to induce the alkaline-specific gene PHR1 and to repress the acid-specific gene PHR2, which both encode cell-surface proteins required for growth and cell polarization (Fonzi, 1999). Although prr2 mutants were able to form hyphae in liquid serum media, they were defective on a solid medium containing serum. Under still weaker induction conditions, such as in some liquid media and on solid Spider medium, the prr 2 mutant was clearly defective in hyphal growth. Thus, Prr2p is needed for the full morphogenetic potential of C. albicans, while PRR 2 overexpression allows filamentation under non-inducing conditions (A. El Barkani \& F. A. Mühlschlegel, unpublished). Prr2p contains a zinc-finger domain that is conserved in the orthologous protein PacC of $A$. nidulans and which is known to recognize the 5'-GCCAAG-3' sequence. Although such sequences occur in the promoter region of the alkaline-induced PHR1 gene they are not required for transcriptional activation of PHR1 (Ramon et al., 1999). It is not yet clear if repression of acid-expressed genes is directly or indirectly regulated by Prr2p. Because the expression of PRR2 depends on the Prr1 protein it was not surprising that prr1 mutants had similar phenotypes to prr2 mutants (Porta et al., 1999). Interestingly, forced expression of one downstream target of the Prr $1 p / \operatorname{Pr} 2 p$ pathway, PHR1, only partially reconstituted the morphogenetic defect of both prr mutants (Porta et al., 1999). This result suggests that components other than Phr1p are involved in morphogenetic control by the Prr2 transcription factor.

\section{Repressing environments and factors}

Besides low $\mathrm{pH}$, low temperature and high cell density, it is known that high glucose concentrations downregulate hyphal development of C. albicans in liquid media. Glucose repression of morphogenesis is also observed initially during growth on solid media, although glucose consumption permits filamentation after several days of growth. On solid media, high osmolarity also inhibits hypha formation (Alex et al., 1998). The presence of easily utilizable nitrogen sources, such as ammonium salts, modulates hyphal development negatively to some degree (Ernst, 2000). In the infected host, inhibition of filamentation by $\gamma$-interferon occurs at contact of C. albicans with lymphocytes (KaloKlein \& Witkin, 1990; Levitz \& North, 1996). Some chemical inhibitors of filamentation are known including diaminobutanone and some antifungals at low concentrations, such as amphotericin and azole antifungals; these compounds inhibit hyphal development by as yet unknown mechanisms (Hawser et al., 1996; Martinez et al., 1990).

The Tup1 transcription factor may be involved in constituting the hypha-repressed state in the presence of glucose and other non-inducing conditions. In $S$. cerevisiae, the Tup1 protein regulates about 60 genes involved in glucose regulation, oxygen stress response and DNA damage. A C. albicans homologue of Tup1p was identified that is $67 \%$ identical to S. cerevisiae Tup1p (Braun \& Johnson, 1997). Tup1p contains seven conserved WD40 repeats at the C terminus, which could anchor Tup1p to some of its DNA-binding proteins, and an N-terminal domain that could interact with a homologue of Ssn6p, as in S. cerevisiae (Keleher et al., 1992; Komachi \& Johnson, 1997). A homozygous C. albicans tup1 mutant grew in filamentous form in all media tested; filaments on most media had the characteristics of pseudohyphae, but in some media had the appearance of true hyphae. Pseudohyphae of a tup1 mutant (unlike pseudohyphae produced by EFG1 overexpression; Stoldt et al., 1997) could not be induced to form germ tubes or true hyphae by the addition of serum (Braun \& Johnson, 1997). Tup1p had activities besides repression of filamentation, because tup 1 mutants failed to grow at $42{ }^{\circ} \mathrm{C}$, grew faster on glycerol and had misshapen cell walls compared to the wild-type. In epistasis experiments most, but not all, of the filamentation phenotype induced by the tup 1 mutation was abolished by the presence of an efg1 mutation, while a $c p h 1$ mutation had very little effect; only a comparison of a tup1 efg1 mutant with a tup1 efg1 cph1 mutant showed a slight influence of the cph1 mutation on hyphal morphogenesis (Braun \& Johnson, 2000). An analysis of transcript levels of hypha-specific genes including HYR1, ALS1, HWP1 and ECE1 showed no differences between $t u p 1$ and $t u p 1 c p h 1$ mutants, only the HWP1 transcript was lowered slightly in the tup1 efg1 cph1 mutant compared to the tup1 efg1 mutant. These results indicate that Efg1p is the main and Cph1p a minor contributor to the tup1 hyphal phenotype. Genes repressed by Tup1p have been identified recently, of which some are expressed in a filament-specific manner (B. Braun \& A. Johnson, unpublished).

In $S$. cerevisiae, the Rap1 protein acts as both a transcriptional silencer and a structural protein at telomeres by binding to a sequence designated the RPG box (Drazinic et al., 1996). A C. albicans protein was identified, Rbf1p, which is not homologous to Rap1p, but binds to the RPG box of $S$. cerevisiae (Ishii et al., 
1997). Rbf1p contains two glutamine-rich regions embedding a region with weak similarity to bHLH domains, which binds to RPG sequences. Homozygous rbf1 null mutants grew in filamentous form in all media tested;the filaments formed had the characteristics of pseudohyphae rather than true hyphae (Ishii et al., 1997; N. Ishii, M. Watanabe \& Y. Aoki, unpublished). Thus, Rbf1p seems to be involved exclusively in pseudohyphal, but not true hyphal growth. In fact, Rbf1p so far seems to be the only component in C. albicans that exclusively determines pseudohyphal growth. On an interesting aside, the authors reported that three alleles of RBF1 were present in the standard disruption strain CAI4. Aneuploidy or triploidy had been previously demonstrated in other C. albicans strains, such as strain SGY243 (Gow et al., 1994; Delbrück et al., 1997), but not in strain CAI4. Besides derepression of filamentous growth, the rbf1 knockout strain showed significantly slower growth and increased sensitivities to high temperature, high osmolarity and hydrogen peroxide compared to the wild-type strain. Virulence of the $r b f 1$ mutant in the mouse model of systemic infection was significantly attenuated (N. Ishii, M. Watanabe \& Y. Aoki, unpublished). Recently, by screening for sequences that mediate Rbf1p-dependent transcriptional regulation, target genes were identified in the heterologous host $S$. cerevisiae. Among the genes identified as Rbf1p targets was the WH11 gene, which in phenotypic switching between a white and an opaque phenotype is specifically expressed in the white phase (Soll, 1997); the level of WH11 transcripts is reduced in homozygous rbf1 mutants compared to wild-type cells (N. Ishii, $M$. Watanabe, S. Fukuchi, M. Arisawa \& Y. Aoki, unpublished). The Sir2 protein (see below) represses hyphal formation, which is consistent with the role of Sir2p as a repressor in S. cerevisiae (although it is unrelated to pseudohyphal growth in this species) (Perez-Martin et al., 1999). Another repressing factor is the Rad6 protein, which besides contributing to UV protection, represses hyphal growth under inducing conditions by an unknown pathway; its deficiency under non-inducing conditions generates a pseudohyphal phenotype (Leng et al., 2000).

\section{Kinases in search of transcriptional pathways}

A number of genes encoding conceptual protein kinases of C. albicans have been identified whose disruption generates a morphogenesis-defective phenotype, but whose input and output pathways are unknown. The SLN1, COS1 and HK1 genes encode possible twocomponent histidine kinases containing sensor and regulator domains. In vitro autophosphorylation activity has been shown for the $S \ln 1 \mathrm{p}$ and Cos1p proteins (Yamada-Okabe et al., 1999). In S. cerevisiae, activation of $\operatorname{Sin} 1 \mathrm{p}$ occurs at normal osmolarity and leads to phosphorylation (and thereby inactivation) of the Ssk1 regulator. Although the C. albicans Sln1 and Ssk1 proteins are the direct homologues of $S$. cerevisiae $S \ln 1$ and Ssk1 proteins, they are not essential in sensing hyperosmolarity. However, hyphal development of $\sin 1$ and ssk1 null mutants is blocked on starvation-type media and is severely impaired on serum agar, while filamentation is normal in all liquid media (Nagahashi et al., 1998; Yamada-Okabe et al., 1999; Calera et al., 2000). Interestingly, while growth of the ssk1 mutant on SLAD medium did not allow formation of hyphae, invasive growth was stimulated significantly compared to the wild-type strain (Calera et al., 2000). A similar phenotype, i.e. a filamentation defect and hyperinvasive growth on solid media, was observed for C. albicans hog1 mutants, which lack a homologue of the $S$. cerevisiae Hog1p MAP kinase, which in this species is a downstream target of Ssk1p (Alonso-Monge et al., 1999). Although to date it has not been resolved if the Sln $1 \mathrm{p}$, Ssk $1 \mathrm{p}$ and Hog $1 \mathrm{p}$ proteins are in a common pathway it can be speculated that Hog $1 \mathrm{p}$ downregulates the MAP kinase pathway responsible for filamentation upstream of Cst20p (Ste20p), as occurs in S. cerevisiae (O'Rourke \& Herskowitz, 1998). Thus, the hyperinvasive characteristics of ssk1 and hog1 mutants is possibly related to activation of the Cph1p transcription factor.

The COS1 (NIK1) and HK1 gene products, which lack transmembrane regions, have no direct homologues in S. cerevisiae. The Cos1 protein is a homologue of the $N$. crassa Nik1 histidine kinase, which in this fungus is involved in hyphal growth and protects against osmotic stress. Null mutants lacking COS1 or HK1 alleles have no defect in osmoprotection, but they are significantly defective in hyphal formation on solid media (starvation-type or medium containing serum), but not in liquid media (Alex et al., 1998; Yamada-Okabe et al., 1999). In addition, the $\mathrm{Hk} 1$ histidine kinase is needed to prevent flocculation of hyphae (Calera \& Calderone, 1999). Interestingly, deletions of SLN1 or COS1 alleles in a $h k 1$ mutant restored filamentation and virulence, suggesting that $S \ln 1 \mathrm{p}$ and Cos1p act upstream of Hk1p, via a negative regulator (Yamada-Okabe et al., 1999). Possibly histidine kinase pathways including the $S \ln 1 \mathrm{p}$ and Cos1p pathways downregulate hyphal development on agar surfaces and within agar.

In S. cerevisiae, one function of the protein kinase C (PKC) pathway is to control the expression of genes encoding cell-wall components and presumably the vectorial transport of secretory vesicles (Banuett, 1998); thus, it could be expected that mutation of the gene encoding the C. albicans PKC homologue would significantly affect morphogenesis. A homozygous $p k c 1$ null strain showed a cell-lysis defect, which was osmotically remediable; however, normal hyphal morphogenesis occurred in stabilized liquid serum media (Paravicini et al., 1996). Because high osmolarity inhibited hyphal formation of wild-type cells on solid media, an effect of the pck1 mutation on Spider media could not be clarified. A gene encoding a downstream target of PKC, the MAP kinase Mkc1p, was also shown not to be absolutely necessary for morphogenesis (NavarroGarcía et al., 1995, 1998). Homozygous mkc1 mutants were less viable and had cell-wall defects, and they were more sensitive to some cell-wall inhibitors. On Spider 
media, hyphal development was blocked, but again, in the presence of serum and other inducers, filamentation occurred.

Cyclin-dependent protein kinases (Cdk) regulate cellcycle progression in eukaryotes. A C. albicans gene (CLN1) encoding a G1-type cyclin homologue has been isolated and both alleles were disrupted (Loeb et al., 1999). Besides slightly retarded growth the mutants were filamentation-defective on solid Spider and serum media; in liquid media an effect was seen only in Spider medium, not with serum as the inducer. The observed filamentation defects were not apparent immediately after inoculation of solid or liquid media, but $\ln 1$ mutants appeared to revert to yeast growth more rapidly than wild-type cells. Although a gene encoding a homologue of the $S$. cerevisiae $\mathrm{Cdc} 28$ protein kinase has been isolated (Sherlock et al., 1994) it is not yet clear if this is the Cdk protein which is activated by $C \ln 1 \mathrm{p}$. Recently, a gene encoding a Cdk homologue CRK1 has been identified, which has a major effect on hyphal growth (J. Chen, S. Zhou, Q. Wang, X. Chen, T. Pan \& H. Liu, unpublished). Hyphal formation under all standard induction conditions, in liquid and on solid media, was severely defective, but not blocked completely. Ectopic expression of CRK1 promoted hyphal growth under non-inducing conditions. Such relatively drastic effects on morphogenesis were hitherto observed only for mutants lacking the Efg1, Ras1 and Tpk2 proteins. There is no information yet on regulators or targets of Crk1p.

\section{Targets of transcription factors}

Several genes have been identified whose expression is induced during (true) hyphal induction. Such genes include the HYR1, ALS3, HWP1 and ECE1 genes, most of which encode cell-wall proteins (Bailey et al., 1996; Birse et al., 1993; Hoyer et al., 1998; Staab et al., 1999). In addition, the EFG1 transcript is transiently downregulated during the initial phases of filamentation (Stoldt et al., 1997). To date there is no evidence that any of the above discussed pathways and transcription factors directly regulate these hypha-specific genes. None of the sequence motifs found in their promoter regions, which have been proposed for the above transcription factors, including an E box (Efg1p), a FRE element (Cph1p) and a PacC element (Prr2p), have yet been proven to be essential for gene regulation. Clearly, the absence of expression of a hypha-specific gene in a filamentation-negative mutant, e.g. lacking the transcription factor Efg1p, could be due to direct or indirect effects on gene regulation. In S. cerevisiae many signalling pathways converge on the promoter of the FLO11 gene, whose gene product is similar to Hwp1p (31\% identity) and the Als protein family (Rupp et al., 1999); recently, it has been shown that the FLO11 promoter is regulated by the heterologous Cph1p, Efg1p and Mcm1p proteins (S. Rupp, unpublished). Expression of HWP1 does not appear to be stringently coupled to hypha formation, since in a prr1 mutant
HWP1 is induced in some media in the absence of filamentation (Porta et al., 1999).

In phenotypic switching (Soll, 1997) there is a link between the ability to form non-yeast growth forms and switching: (1) smooth colonies are composed only of yeast-form cells, while wrinkled colonies mainly contain branched hyphae and other colony types are a mixture of yeast cells, hyphae and/or pseudohyphal cells; (2) in the white-opaque switching of strain WO-1 and its derivatives, the opaque cells are elongated or rod-like and are unable to form true hyphae, while the whitephase cells have a yeast-cell form and are competent to form hyphae; (3) deletion of the Sir2 protein enhances hypha formation and increases phenotypic switching, while it also increases chromosomal recombination (Soll et al., 1993; Perez-Martín et al., 1999). To date it is unclear whether Sir $2 p$ is actually involved in 'natural' switching, or if a general increase in chromosomal recombination is the predominant cause of switch phenotypes (Janbon et al., 1999; Rustchenko et al., 1994). Recent results implicate the Efg1p transcription factor in white-opaque phenotypic switching, because (1) the major EFG1 transcript is expressed specifically in white-type, not in the opaque-type cells, (2) low EFG1 expression levels generate an opaque-like cellular phenotype in strain CAI8, which is not known to switch between a white and an opaque form and (3) overexpression of EFG1 forces opaque-type cells to switch to the white form (Sonneborn et al., 1999a). In support of these data it was recently reported that efg1 null mutants derived from strain WO-1 have the elongated cell morphology and colony phenotype typical of opaque cells under all conditions and are unable to switch spontaneously to the alternate white phenotype, although other switching phenotypes are Efg1p-independent (Srikantha et al., 2000). As in the case of dimorphism, an Efg1p-target gene in phenotypic switching is not yet known. Likewise, the role of Efg1p in chlamydospore formation needs to be established, for whose morphogenesis it is the only defined factor (Sonneborn et al., 2000).

\section{Saccharomyces as a model of Candida morphogenesis?}

Although Sacch. cerevisiae is an important eukaryotic model organism, homologous components in other fungi, including Schizosaccharomyces pombe and C. albicans, do not always have identical functions. Furthermore, some morphogenetic phenotypes of $C$. albicans such as true hypha formation, phenotypic switching and chlamydospore formation are not observed in Sacch. cerevisiae, which on the other hand is able to develop meiospores. Virulence factors regulating the interaction with host cells or the immune system are relevant for the biology of C. albicans as a pathogen, not of Sacch. cerevisiae.

On the first look, as detailed above, conserved MAP kinase and cAMP/PKA pathways function in identical morphogenetic processes in $S$. cerevisiae as in $C$. 
albicans. The C. albicans Cst20p/Hst7p/Cek1p/Cph1p pathway corresponds to the $S$. cerevisiae Ste20p/Ste7/ Kss1p/Ste12p pathway with regard to structures and sequential functions of its components (Liu et al., 1993; Madhani \& Fink, 1997). Although knowledge of the C. albicans cAMP/PKA pathway is still rudimentary, the Ras1/Tpk2/Efg1 components have structurally homologous counterparts in the $S$. cerevisiae Ras2/Tpk2/ Phd1 (Sok2) proteins (Gimeno et al., 1992; Gimeno \& Fink, 1994; Robertson \& Fink, 1998; Ward et al., 1995). However, besides the different morphogenetic outputs, both pathways do not seem equally important in directing morphogenesis in C. albicans, as in $S$. cerevisiae. In S. cerevisiae, defects in the MAP kinase pathway have a major effect on pseudohypha formation, while corresponding deficiencies in C. albicans affect morphogenesis only under special conditions, but not during induction by serum and other inducers. The relative importance of the MAP kinase pathway in $S$. cerevisiae may be related to the fact that some of its components are used in signalling leading to mating, which is a process unknown for C. albicans (although recently a mating-type-like locus has been described; Hull \& Johnson, 1999). Although the Ras and PKA isoforms appear functionally equivalent in both fungal species, the downstream APSES proteins Efg1p and Phd1p do not seem to represent functional homologues. The function of Phd1p in S. cerevisiae is clearly detected only in phd1 ste 12 double mutants, which are more defective than the ste 12 mutant in pseudohyphal growth. In C. albicans efg1 mutants, the formation of true hyphae is blocked almost completely, while the ability to form pseudohyphae under some conditions remains and is even derepressed (opaque-like phenotype), at least in some genetic backgrounds (Lo et al., 1997; Stoldt et al., 1997). The combination of efg1 and cph1 mutations in a single strain reduces pseudohyphal growth (although only partially in our hands), but formation of true hyphae is blocked, as in the efg1 single mutant. On the other hand, both mutants show florid hypha formation under microaerophilic/embedded conditions (see above). The latter pathway is not known in S. cerevisiae, but makes sense in C. albicans, which allows it to form filaments in host niches lacking serum or other inducers.

In summary, although C. albicans research on dimorphism has profited and will continue to profit from knowledge obtained in S. cerevisiae, functions of homologous components cannot be equated. In fact, understanding the differences between the two fungal species will be most important to establish the reasons for the particular virulence of C. albicans.

\section{Future directions}

The current knowledge on the mechanisms of yeasthyphal transitions in C. albicans can be characterized by the statement 'many components but few connections'. Therefore, one would wish that efforts should be directed more towards an analysis of the functional linkages instead of an expansion of the list of components affecting morphogenesis. Much of the latter research is driven by the exploration of genes/ components with homologous counterparts in $S$. cerevisiae. This is a valid approach, but results should be interpreted with caution and will not necessarily clarify the particular virulence features of C. albicans. Most of the about 20 established components involved in dimorphism have no effect in stringent conditions, such as in liquid induction media, but only on solid media after days of growth, in which environmental parameters are changing. In this case the typical 'end-point analysis' should at least be complemented by a temporal analysis of filamentation, taking into account the switch back of (pseudo-) hyphal to yeast growth occurring under this condition. A strong appeal must be made to be absolutely clear about the types of filamentous growths that are monitored. A clear distinction between hyphae and pseudohyphae is not always made in the literature; the designation of both types of filaments as hyphae muddies the water and may deter new researchers from entering this fascinating area of research.

The mechanisms of chlamydospore formation and of spontaneous phenotypic switching are two of the most intriguing areas of research on C. albicans biology in the future, which are still in their infancies. As for dimorphism, the presence and the functions of signalling pathways need to be pursued, not only regarding the yeast-hyphae transition, but also regarding the reverse transition; in addition, input and output signals of morphogenetic pathways need to be clarified. Much of the research in the near future will be driven by genomic approaches, which will identify genome-wide expression targets by array techniques. Furthermore, sensors responding to environmental changes, especially in the host during infection, need to be identified, as well as their linkages to downstream signalling pathways.

\section{Acknowledgements}

I am most grateful to my colleagues B. Braun, A. Brown, R. Calderone, G. Fink, B. Fonzi, N. Ishii, J. Köhler, C. Kumamoto, H. Liu, F. Mühlschlegel, J. Pla, S. Rupp, D. Soll and K. Schroeppel for useful comments and communication of unpublished results. I thank A. Sonneborn and D. Bockmühl for useful criticisms.

\section{References}

Alex, L. A., Korch, C., Selitrennikoff, C. P. \& Simon, M. I. (1998). COS1, a two-component histidine kinase that is involved in hyphal development in the opportunistic pathogen Candida albicans. Proc Natl Acad Sci USA 95, 7069-7073.

Alonso-Monge, R., Navarro-García, F., Molero, G., Diez-Orejas, R., Gustin, M., Pla, J., Sánchez, M. \& Nombela, C. (1999). Role of the mitogen-activated protein kinase Hog $1 \mathrm{p}$ in morphogenesis and virulence of Candida albicans. J Bacteriol 181, 3058-3068.

Aramayo, R., Peleg, Y., Addison, R. \& Metzenberg, R. (1996). Asm- $1^{+}$, a Neurospora crassa gene related to transcriptional regulators of fungal development. Genetics 144, 991-1003. 
Bailey, D. A., Feldmann, P. J. F., Bovey, M., Gow, N. A. R. \& Brown, A. J. P. (1996). The Candida albicans HYR1 gene, which is activated in response to hyphal development, belongs to a gene family encoding yeast cell wall proteins. J Bacteriol 178, 5353-5360.

Banuett, F. (1998). Signalling in yeasts: an informational cascade with links to the filamentous fungi. Microbiol Mol Biol Rev 62, 249-274.

Bernards, R. (1995). Flipping the Myc switch. Curr Biol 5, 859-861.

Birse, C. E., Irwin, M. Y., Fonzi, W. A. \& Sypherd, P. S. (1993). Cloning and characterization of ECE1, a gene expressed in association with cell elongation of the dimorphic pathogen Candida albicans. Infect Immun 61, 3648-3655.

Braun, B. R. \& Johnson, A. D. (1997). Control of filament formation in Candida albicans by the transcriptional repressor TUP1. Science 277, 105-109.

Braun, B. R. \& Johnson, A. D. (2000). TUP1, CPH1 and EFG1 make independent contributions to filamentation in Candida albicans. Genetics 155, 57-67.

Brown, D. H., Jr, Giusani, A. D., Chen, X. \& Kumamoto, C. (1999). Filamentous growth of Candida albicans in response to physical environmental cues and its regulation by the unique $C Z F 1$ gene. Mol Microbiol 34, 651-662.

Buffo, J., Herman, M. A. \& Soll, D. R. (1984). A characterization of $\mathrm{pH}$-regulated dimorphism in Candida albicans. Mycopathologia $85,21-30$.

Calera, J. A. \& Calderone, R. (1999). Flocculation of hyphae is associated with a deletion in the putative CaHK1 two-component histidine kinase gene from Candida albicans. Microbiology 145, 1431-1442.

Calera, J. A., Zhao, X. J. \& Calderone, R. (2000). Defective hyphal development and avirulence caused by a deletion of the SSK1 response regulator gene in Candida albicans. Infect Immun 68, 518-525.

Chaffin, W. L. (1984). Site selection for bud and germ tube emergence in Candida albicans. J Gen Microbiol 130, 431-440.

Chant, J. (1994). Cell polarity in yeast. Trends Genet 10, 328-333.

Cormack, B. P., Bertram, G., Egerton, M., Gow, N. A. R., Falkow, S. \& Brown, A. J.P. (1997). Yeast-enhanced green fluorescent protein (yEGFP): a reporter of gene expression in Candida albicans. Microbiology 143, 303-311.

Csank, C., Makris, C., Meloche, S., Schröppel, K., Röllinghoff, M., Dignard, D., Thomas, D. Y. \& Whiteway, M. (1997). Derepressed hyphal growth and reduced virulence in a VH1 family-related protein phosphatase mutant of the human pathogen Candida albicans. Mol Biol Cell 8, 2539-2551.

Csank, C., Schröppel, K., Leberer, E., Harcus, D., Mohamed, O., Meloche, S., Thomas, D. Y. \& Whiteway, M. (1998). Roles of the Candida albicans mitogen-activated protein kinase homolog, Cek1p, in hyphal development and systemic candidiasis. Infect Immun 66, 2713-2721.

Delbrück, S. \& Ernst, J. F. (1993). Morphogenesis-independent regulation of actin transcript levels in the pathogenic yeast Candida albicans. Mol Microbiol 10, 859-866.

Delbrück, S., Sonneborn, A., Gerads, M., Grablowitz, A. H. \& Ernst, J. F. (1997). Characterization and regulation of the genes encoding ribosomal proteins L39 and S7 of the human pathogen Candida albicans. Yeast 13, 1199-1210.

Drazinic, C. M., Smerage, J. B., Lopez, M. C. \& Baker, H. (1996). Activation mechanism of the multifunctional transcription factor repressor-activator protein 1. Mol Cell Biol 16, 3187-3196.
Dutton, J. R., Johns, S. \& Miller, B. L. (1997). StuAp is a sequencespecific transcription factor that regulates developmental complexity in Aspergillus nidulans. EMBO J 16, 5710-5721.

Ernst, J. F. (2000). Regulation of dimorphism in Candida albicans. In Contributions to Microbiology, vol. 5, Dimorphism in Human Pathogenic and Apathogenic Yeasts, pp. 98-111. Edited by J. F. Ernst \& A. Schmidt. Basel: Karger.

Facchini, L. M., Chen, S., Marhin, W. W., Lear, J. N. \& Penn, L. Z. (1997). The Myc negative autoregulation mechanism requires Myc-Max association and involves the $c$-myc P2 minimal promoter. Mol Cell Biol 17, 100-114.

Feng, Q., Summers, E., Guo, B. \& Fink, G. (1999). Ras signalling is required for serum-induced hyphal differentiation in Candida albicans. J Bacteriol 181, 6339-6346.

Fonzi, W. A. (1999). PHR 1 and PHR 2 of Candida albicans encode putative glycosidases required for proper cross-linking of $\beta-1,3-$ and $\beta$-1,6-glucans. J Bacteriol 181, 7070-7079.

Fonzi, W. A. \& Irwin, M. Y. (1993). Isogenic strain construction and gene mapping in Candida albicans. Genetics 134, 717-728.

Gimeno, C. J. \& Fink, G. R. (1994). Induction of pseudohyphal growth by overexpression of PHD1, a Saccharomyces cerevisiae gene related to transcriptional regulators of fungal development. Mol Cell Biol 14, 2100-2112.

Gimeno, C. J., Ljungdahl, P. O., Styles, C. A. \& Fink, G. R. (1992). Unipolar cell divisions in the yeast $S$. cerevisiae lead to filamentous growth: regulation by starvation and RAS. Cell 68, 1077-1090.

Gow, N. A. R., Robbins, P. W., Leister, J. W., Brown, A. J. P., Fonzi, W. A., Chapman, T. \& Kinsman, O. S. (1994). A hyphalspecific chitin synthase gene (CHS2) is not essential for growth, dimorphism, or virulence of Candida albicans. Proc Natl Acad Sci USA 91, 6216-6220.

Hawser, S., Francolini, M. \& Islam, K. (1996). The effects of antifungal agents on the morphogenetic transformation by Candida albicans in vitro. J Antimicrob Chemother 38, 579-587.

Hoyer, L. L., Payne, T. L., Bell, M., Myers, A. M. \& Scherer, S. (1998). Candida albicans ALS3 and insights into the nature of the ALS gene family. Curr Genet 33, 451-459.

Hube, B. (1996). Candida albicans secreted aspartyl proteinases. Curr Top Med Mycol 7, 55-69.

Hull, C. M. \& Johnson, A. D. (1999). Identification of a mating type-like locus in the asexual pathogenic yeast Candida albicans. Science 285, 1271-1275.

Ishii, N., Yamamoto, M., Yoshihara, F., Arisawa, M. \& Aoki, Y. (1997). Biochemical and genetic characterization of Rbf1p, a putative transcription factor of Candida albicans. Microbiology 143, 429-435.

Janbon, G., Sherman, F. \& Rustchenko, E. (1999). Appearance and properties of L-sorbose-utilizing mutants of Candida albicans obtained on a selective plate. Genetics 153, 653-664.

Joshi, K. R., Solanki, A. \& Prakash, P. (1993). Morphological identification of Candida species on glucose agar, rice extract agar and corn meal agar with and without Tween-80. Indian J Pathol Microbiol 36, 48-52.

Kalo-Klein, A. \& Witkin, S. S. (1990). Prostaglandin $\mathrm{E}_{2}$ enhances and gamma interferon inhibits germ tube formation in Candida albicans. Infect Immun 58, 260-262.

Keleher, C. A., Redd, M. J., Schultz, J., Carlson, M. \& Johnson, A. D. (1992). Ssn6-Tup1 is a general repressor of transcription in yeast. Cell 68, 709-719.

Köhler, J. R. \& Fink, G. R. (1996). Candida albicans strains heterozygous and homozygous in mitogen-activated protein 
kinase signalling components have defects in hyphal development. Proc Natl Acad Sci USA 93, 13223-13228.

Komachi, K. \& Johnson, A. D. (1997). Residues in the WD repeats of Tup1 required for interaction with alpha2. Mol Cell Biol 17, 6023-6028.

Leberer, E., Harcus, D., Broadbent, I. D. \& 7 other authors (1996). Signal transduction through homologs of the Ste20p and Ste7p protein kinases can trigger hyphal formation in the pathogenic fungus Candida albicans. Proc Natl Acad Sci USA 93, 1321713222.

Leng, P., Sudbery, P. E. \& Brown, A. J. P. (2000). Rad6p represses yeast-hypha morphogenesis in the human fungal pathogen, Candida albicans. Mol Microbiol 35, 1264-1275.

Leuker, C. E., Hahn, A.-M. \& Ernst, J. F. (1992). $\beta$-Galactosidase of Kluyveromyces lactis (Lac4p) as reporter of gene expression in Candida albicans and C. tropicalis. Mol Gen Genet 235, 235-241.

Levitz, S. M. \& North, E. A. (1996). Gamma interferon gene expression and release in human lymphocytes directly activated by Cryptococcus neoformans and Candida albicans. Infect Immun 64, 1595-1599.

Liu, H., Styles, C. A. \& Fink, G. R. (1993). Elements of the yeast pheromone response pathway required for filamentous growth of diploids. Science 262, 1741-1744.

Liu, H., Köhler, J. \& Fink, G. R. (1994). Suppression of hyphal formation in Candida albicans by mutation of a STE12 homolog. Science 266, 1723-1725.

Lo, H.-J., Köhler, J. R., Didomenico, B., Loebenberg, D., Cacciapuoti, A. \& Fink, G. R. (1997). Nonfilamentous C. albicans mutants are avirulent. Cell 90, 939-949.

Loeb, J. D. J., Sepulveda-Becerra, M., Hazan, I. \& Liu, H. (1999). A $G_{1}$ cyclin is necessary for maintenance of filamentous growth in Candida albicans. Mol Cell Biol 19, 4019-4027.

Madhani, H. D. \& Fink, G. R. (1997). Combinatorial control required for the specificity of yeast MAPK signalling. Science $\mathbf{2 7 5}$, 1314-1317.

Martinez, J. P., Lopez-Ribot, J. L., Gil, M. L., Sentandreu, R. \& Ruiz-Herrera, J. (1990). Inhibition of the dimorphic transition of Candida albicans by the ornithine decarboxylase inhibitor 1,4diaminobutanone: alterations in the glycoprotein composition of the cell wall. J Gen Microbiol 136, 1937-1943.

Miller, K. Y., Wu, J. \& Miller, B. L. (1992). StuA is required for cell pattern formation in Aspergillus. Genes Dev 6, 1770-1782.

Mösch, H.-U., Roberts, R. L. \& Fink, G. R. (1996). Ras2 signals via the $\mathrm{Cdc} 42 / \mathrm{Ste} 20 /$ mitogen-activated protein kinase module to induce filamentous growth in Saccharomyces cerevisiae. Proc Natl Acad Sci USA 93, 5352-5356.

Montazeri, M. \& Hedrick, H. G. (1984). Factors affecting spore formation in a Candida albicans strain. Appl Environ Microbiol 47, 1341-1342.

Morschhäuser, J., Michel, S. \& Staib, P. (1999). Sequential gene disruption in Candida albicans by FLP-mediated site-specific recombination. Mol Microbiol 32, 547-556.

Nagahashi, S., Mio, T., Ono, N., Yamada-Okabe, T., Arisawa, M., Bussey, H. \& Yamada-Okabe, H. (1998). Isolation of CaSLN1 and CaNIK1, the genes for osmosensing histidine kinase homologues, from the pathogenic fungus Candida albicans. Microbiology 144, 425-432.

Navarro-García, F., Sánchez, M., Pla, J. \& Nombela, C. (1995). Functional characterization of the MKC1 gene of Candida albicans, which encodes a mitogen-activated protein kinase homolog related to cell integrity. Mol Cell Biol 15, 2197-2206.
Navarro-García, F., Alonso-Monge, R., Rico, H., Pla, J., Sentandreu, R. \& Nombela, C. (1998). A role for the MAP kinase gene $M K C 1$ in cell wall construction and morphological transitions in Candida albicans. Microbiology 144, 411-424.

Niimi, M. (1996). Dibutyryl cyclic AMP-enhanced germ tube formation in exponentially growing Candida albicans cells. Fungal Genet Biol 20, 79-83.

Niimi, M., Niimi, K., Tokunaga, J. \& Nakayama, H. (1980). Changes in cyclic nucleotide levels and dimorphic transition in Candida albicans. J Bacteriol 142, 1010-1014.

Odds, F. C. (1988). Candida and Candidosis, 2nd edn. London: Baillière Tindall.

O'Rourke, S. M. \& Herskowitz, I. (1998). The Hog1 MAPK prevents cross talk between the HOG and pheromone response MAPK pathways in Saccharomyces cerevisiae. Genes Dev 12, 2874-2886.

Pan, X. \& Heitman, J. (1999). Cyclic AMP-dependent protein kinase regulates pseudohyphal differentiation in Saccharomyces cerevisiae. Mol Cell Biol 19, 4874-4887.

Paravicini, G., Menoza, A., Antonsson, B., Cooper, M., Losberger, C. \& Payton, M. A. (1996). The Candida albicans PCK1 gene encodes a protein kinase $\mathrm{C}$ homolog necessary for cellular integrity but not dimorphism. Yeast 12, 741-756.

Perez-Martin, J., Uria, J. A. \& Johnson, A. D. (1999). Phenotypic switching in Candida albicans is controlled by the SIR2 gene. EMBO J 18, 2580-2592.

Porta, A., Ramon, A. M. \& Fonzi, W. A. (1999). PRR1, the homolog of Aspergillus nidulans palF, controls pH-dependent gene expression and filamentation in Candida albicans. J Bacteriol 181, 7516-7523.

Rademacher, F., Kehren, V., Stoldt, V. R. \& Ernst, J. F. (1998). A Candida albicans chaperonin subunit $(\mathrm{CaCct} 8 \mathrm{p})$ as a suppressor of morphogenesis and Ras phenotypes in C. albicans and Saccharomyces cerevisiae. Microbiology 144, 2951-2960.

Ramon, A. M., Porta, A. \& Fonzi, W. A. (1999). Effect of environmental $\mathrm{pH}$ on morphological development of Candida albicans is mediated via the PacC-related transcription factor encoded by PRR2. J Bacteriol 181, 7524-7530.

Riggle, P. J., Andrutis, K. A., Chen, X., Tzipori, S. R. \& Kumamoto, C. (1999). Invasive lesions containing filamentous forms produced by a Candida albicans mutant that is defective in filamentous growth in culture. Infect Immun 67, 3649-3652.

Ritz, K. \& Crawford, J. W. (1999). Colony development in nutritionally heterogeneous environments. In The Fungal Colony, pp. 49-74. Edited by N. A. R. Gow, G. D. Robson \& G. M. Gadd. Cambridge: Cambridge University Press.

Robertson, L. S. \& Fink, G. R. (1998). The three A kinases have specific signalling functions in pseudohyphal growth. Proc Natl Acad Sci USA 95, 13783-13787.

Rupp, S., Summers, E., Lo, H.-J., Madhani, H. \& Fink, G. (1999). MAP kinase and cAMP filamentation signalling pathways converge on the unusually large promoter of the yeast FLO11 gene. EMBO J 18, 1257-1269.

Rustchenko, E., Howard, D. H. \& Sherman, F. (1994). Chromosomal alterations of Candida albicans are associated with the gain and loss of assimilating functions. J Bacteriol 176, 3231-3241.

Sabie, F. T. \& Gadd, G. M. (1992). Effect of nucleosides and nucleotides and the relationship between cellular adenosine $3^{\prime}: 5^{\prime}$ cyclic monophosphate (cyclic AMP) and germ tube formation in Candida albicans. Mycopathologia 119, 147-156.

Santos, M. A., Perreau, V. M. \& Tuite, M. F. (1996). Transfer RNA 
structural change is a key element in the reassignment of the CUG codon in Candida albicans. EMBO J 15, 5060-5068.

Scherer, S. \& Magee, P. T. (1990). Genetics of Candida albicans. Microbiol Rev 54, 226-241.

Sherlock, G., Bahman, A. M., Mahal, A., Shieh, J. C., Ferreira, M. \& Rosamond, J. (1994). Molecular cloning and analysis of CDC28 and cyclin homologues from the human fungal pathogen Candida albicans. Mol Gen Genet 245, 716-723.

Soll, D. R. (1997). Gene regulation during high-frequency switching in Candida albicans. Microbiology 143, 279-288.

Soll, D. R., Morrow, B. \& Srikantha, T. (1993). High-frequency phenotypic switching in Candida albicans. Trends Genet 9, 61-65.

Sonneborn, A., Tebarth, B. \& Ernst, J. F. (1999a). Control of whiteopaque phenotypic switching in Candida albicans by the Efg1p morphogenetic regulator. Infect Immun 67, 4655-4660.

Sonneborn, A., Bockmühl, D. \& Ernst, J. F. (1999b). Chlamydospore formation in Candida albicans requires the Efg1p morphogenetic regulator. Infect Immun 67, 5514-5517.

Sonneborn, A., Bockmühl, D. P., Gerads, M., Kurpanek, K., Sanglard, S. \& Ernst, J. F. (2000). Protein kinase A encoded by TPK 2 regulates dimorphism of Candida albicans. Mol Microbiol 35, 386-396.

Srikantha, T., Klapach, A., Lorenz, W. W., Tsai, L. K, Laughlin, L. A., Gorman, J. A. \& Soll, D. R. (1996). The sea pansy Renilla reniformis luciferase serves as a sensitive bioluminescent reporter for differential gene expression in Candida albicans. J Bacteriol 178, 121-129.

Srikantha, T., Tsai, L. K., Daniels, K. \& Soll, D. R. (2000). EFG1 null mutants of Candida albicans switch, but cannot express the complete phenotype of white-phase budding cells. J Bacteriol $\mathbf{1 8 2}$, 1580-1591.

Staab, J. F., Bradway, S. D., Fidel, P. L. \& Sundstrom, P. (1999). Adhesive and mammalian transglutaminase substrate properties of Candida albicans Hwp1p. Science 283, 1535-1538.

Stoldt, V. R., Sonneborn, A., Leuker, C. \& Ernst, J. F. (1997). Efg1, an essential regulator of morphogenesis of the human pathogen Candida albicans, is a member of a conserved class of bHLH proteins regulating morphogenetic processes in fungi. EMBO J 16, 1982-1991.

Ward, M. P., Gimeno, C. J., Fink, G. R. \& Garrett, S. (1995). SOK2 may regulate cyclic AMP-dependent protein kinase-stimulated growth and pseudohyphal development by repressing transcription. Mol Cell Biol 15, 6854-6863.

Wilson, R. B., Davis, D. \& Michell, A. P. (1999). Rapid hypothesis testing with Candida albicans through gene disruption with short homology regions. J Bacteriol 181, 1868-1874.

Yamada-Okabe, T., Mio, T., Ono, N., Kashima, Y., Matsui, M., Arisawa, M. \& Yamada-Okabe, Y. (1999). Roles of three histidine kinase genes in hyphal development and virulence of the pathogenic fungus Candida albicans. J Bacteriol 181, 7243-7247. 